\title{
Análise dos sintomas e das alterações otológicas em pacientes com sintomatologia dolorosa na articulação temporomandibular
}

\author{
Analysis of symptoms and otologic alterations in patients with symptomatology \\ painful in joint temporomandibular
}

Francisca Laura Ferreira de Sousa ${ }^{1}$; Rachel Costa Façanha ${ }^{2}$

\begin{abstract}
Resumo: A disfunção temporomandibular (DTM) é um conjunto de sinais e sintomas, que prejudica as funções de articulação da fala, de mastigação, respiração, e muitas vezes encontra-se associada asintomatologia auditiva. Essa pesquisa teve como objetivo investigar a relação da dor na articulação temporomandibular com a presença de sintomas e alterações otológicas por meio de um estudo analítico, observacional do tipo transversal, em 25 pacientes com dor causada por processo inflamatório articular decorrente de disfunção temporomandibular, de ambos os sexos, maior de idade, atendidos na Clínica Escola de Fonoaudiologia da Universidade Ceuma. Dos 25 pacientes, 88\% referiram pelo menos um sintoma otológico.O zumbido foi referido em $72 \%$ dos casos, a otalgiaem $56 \%$ e a sensação de plenitude auricular em 52\%. Os achados deste estudo apontam alto percentual de sintomas otológicos em pacientes com sinais e sintomas de DTM, destacando-se a necessidade de estudos e pesquisas mais complexos e com uma amostra maior pra comprovar as específicas relações causaefeito entre ambos.
\end{abstract}

Palavras-chave: Articulação Temporomandibular; Perda auditiva; Dor.

Abstrat: The temporomandibular disorders (TMD) is a set of signs and symptoms, which affect the joint functions of speech, chewing, breathing, and often is associated with auditory symptoms. This research aimed to investigate the relationship of pain in the temporomandibular joint with the presence of symptoms and ear changes via an analytical observational cross-sectional study in 25 patients with pain caused by inflammation joint due to temporomandibular dysfunction, of both sexes, of legal age, assisted at the Clinical School of Speech Therapy at the University Ceuma. Of the 25 patients, $88 \%$ reported at least one otologic symptoms. Tinnitus has been reported in $72 \%$ of cases, otalgia $56 \%$ and ear fullness of $52 \%$. The findings of this study indicate a high percentage of otologic symptoms in patients with signs and symptoms of TMD, highlighting the need for studies and research more complex and a larger sample to confirm the specific cause-effect relationships between them.

Keywords: Temporomandibular Joint; Auditiva loss; Ache.

\footnotetext{
${ }^{1}$ Graduado do curso de Fonoaudiologia - UNICEUMA

${ }^{2}$ Docentes da Universidade Ceuma
} 


\section{Introdução}

A articulação temporomandibular, mais conhecida como ATM, é uma articulação de grande complexidade. Essa articulação realiza de 1500 a 2000 movimentos diariamente. Sua ação é atuar na mastigação, na fala e inclusive no simples fato de abertura da boca como no ato do bocejo. A disfunção temporomandibular (DTM) é um conjunto de sinais e sintomas tais como dor, sons nas articulações temporomandibulares e movimentos mandibulares restritos ${ }^{1}$. Segundo Felício ${ }^{1}$, prejudica as funções de articulação da fala, de mastigação, respiração, e muitas vezes encontrase associada à sintomatologia auditiva.

As possíveis modificações nas estruturas da ATM também são preocupações do fonoaudiólogo, pois atingem diretamente 0 sistema estomatognático (SE) e suas funções. $O$ equilíbrio das estruturas e as funções do sistema estomatognático dependem do adequado funcionamento da Articulação Temporomandibular, que precisa da oclusão dental correta para a realização das funções ao movimentar a mandíbula. O desequilíbrio poderá acarretar em uma disfunção dessa articulação e causar diversos sinais e sintomas como, manifestações auditivas do tipo zumbido e otalgia ${ }^{\Upsilon}$.

Alterações na musculatura mastigatória por causa da DTM podem causar, como consequência, disfunção da tuba auditiva, pressão nos ouvidos, desequilíbrio, perda da audição, otalgia, zumbido e cefaléia. Isso acontece devido à grande proximidade anatômica e funcional entre as estruturas da orelha e a ATM, incluindo a inervação e vascularização ${ }^{2}$.

É comum pacientes com dor na ATM também se queixarem de dor no ouvido. O ouvido encontra-se contido no osso temporal e relaciona-se com o côndilo mandibular, separado deste apenas pela parede timpânica ${ }^{3}$.

Malklin ${ }^{4}$ relata que os sintomas otológicos mais comumente associados à otalgia e a DTM é a percepção de zumbido e a plenitude auricular, podendo ser explicadas pelo deslocamento da cabeça da mandíbula para posterior e distal, o que produz pressão direta sobre o nervo aurículo-temporal, sobre as estruturas da orelha e especialmente sobre a tuba auditiva. Encontrado-se também com grande frequência sintomas como otalgia (na maioria dos estudos), vertigem e tontura em pacientes portadores destas alterações articulares, prevalecendo em indivíduos do sexo feminino.

A relação entre DTM e sintomas otológicos tem sido explicada com base na relação embriológica, anatômica e funcional da região que compreende as ATMs, a musculatura inervada pelo trigêmio e as estruturas da orelha média ${ }^{4}$. Dentre outras coisas, tem sido sugerido que as alterações musculares em pacientes com DTM, como o espasmo do músculo pterigóideo lateral, levam à hipertonia do músculo tensor do tímpano, causando alterações no ciclo de abertura da tuba auditiva e consequente à redução na ventilação da orelha média ${ }^{5}$. No entanto, alguns autores contestam esta hipótese $e^{6,7,8}$.

Um dos exames realizados para o diagnóstico de perda auditiva é a audiometria tonal limiar, que quantifica por frequência os limiares auditivos. Estes estando maiores que 25 dBNA, são categorizados como indivíduos com déficit na audição. O exame realizado para a verificação da orelha média é a imitanciometria, que analisa a curva timpanométrica, categorizada como do tipo "A" para sujeitos sem alteração nesta parte da orelha, e os reflexos acústicos em resposta a condição do músculo estapediano ${ }^{10,9}$. 
Há uma quantidade restrita de estudos que enfatizam a alteração auditiva nas DTMs. Logo, faz-se necessária a realização de estudos que mostrem a importância de se relacionar alteração auditiva e o grau de dor na DTM e a atuação integral do profissional fonoaudiólogo nesta disfunção e a importância da atuação multiprofissional. Com base nesse argumento, o propósito deste trabalho foi encontrar uma inter-relação entre as alterações e sintomas auditivos e a dor na DTM.

\section{Material e Métodos}

Esta pesquisa trata-se de um estudo analítico, observacional do tipo transversal, quantitativo. Realizado na Clínica Escola de Fonoaudiologia da Universidade Ceuma, na cidade de São Luís, estado do Maranhão.A amostra foialeatória simples composta por 25 pacientes que apresentavam sinais e sintomas para a presença sugestiva de DTM, a pesquisa foi realizada no período de abril a outubro de 2015.

Foram incluídos indivíduos de ambos os sexos, maior de idade, que procuraram 0 serviço de Fonoaudiologia da Clínica Escola da Universidade Ceuma. Os pacientes participaram do estudo de forma consecutiva, à medida que foram atendidos no serviço, desde que os mesmos cumprissem os critérios de inclusão e aceitassem participar da pesquisa. $O$ paciente deveria apresentar dor na articulação temporomandibular durante a função mandibular e a palpação das estruturas, com limitação ou desvios nos movimentos mandibulares, ruídos na ATM, e relação oclusal estática ou dinâmica anormal, sem ter realizado tratamento para articulação temporomandibular.
A coleta foi realizada pela própria pesquisadora através de uma anamnese e avaliação clínica da articulação temporomandibular, antes de realizarem tratamento para DTM. O protocolo desta pesquisa foi montado baseando-se em protocolos preexistentes validados de autores como Fonseca ${ }^{12}$, Manfredi e Vendite ${ }^{5}$ e Bianchini ${ }^{8}$. A investigação deteve-se aos sinais e sintomas de DTM e padrão dos movimentos mandibulares.

A entrevista (anamnese) procurou caracterizar o paciente com relação à idade, sexo, sinais e sintomas de DTM e otológicos.

$\mathrm{Na}$ anamnese continha as seguintes variáveis: Presença de queixa referente à abertura de boca como dificuldade, dor ou desconforto, percepção de estalido ${ }^{5}$; Percepção do paciente quanto a presença de dor ou desconforto muscular e/ou articular quando ele realizava a abertura de boca $^{14}$; Presença de queixa relativa a sintomatologia otológica (otalgia, plenitude auricular, zumbido, tontura, hipoacusia) e de cefaleia9; Presença de dificuldade, dor ou cansaço ou ambos ao executar funções estomatognáticas como mastigar, falar e bocejar ${ }^{5}$.

No que tange a avaliação da dor na ATM, seráempregadoo escore de 0 a 3 segundoGracely ${ }^{15}$, onde 0 -não há presença de dor, 1 - dor fraca, 2 - dor moderada, 3 - dor fortecom retirada protetiva. A palpação foi realizada em regiãoretrodiscaldurante a abertura oral máxima e em região capsular com a mandíbula em repouso palpando a áreapré-auricular(na posição do côndilo) ${ }^{15}$.

Após a entrevista os pacientes foram encaminhados para a Clínica Escola de Audiologia da Universidade Ceuma, para a realização de anamnese audiológica e de exames auditivos, que incluíram a meatoscopia (inspeção do meato auditivo externo), 
audiometria tonal limiar por via aérea e óssea e índice de reconhecimento de fala (IPRF), através de equipamento calibrado da marca Interacoustic Ad229, e a medida da imitância acústica (timpanometria e pesquisa do reflexo acústico), por meio do equipamento AT235h.

Em cumprimento aos requisitos exigidos pela Resolução 466/12 do Conselho Nacional de Saúde, a pesquisa foi submetida e aprovada pelo Comitê de Ética em Pesquisa da Universidade Ceuma, com o CAAE de número 48717615.9.0000.5084.Os pacientes receberam todas as explicações necessárias ao entendimento dos objetivos e suas consequências, conforme apresentado no Termo de Consentimento Livre e Esclarecido.Os dados foram descritos por meio de frequência relativa em valor percentual, associando a presença de intensidade da dor com achados otológicos, expostos por meio de tabelas.

\section{Resultados e Discussão}

Foram avaliados 25 pacientes
que procuraram o serviço de
Audiologia da Universidade Ceuma, com uma média de 31,75 anos de idade.Entre eles, 32\% tinham entre 18 e 25 anos, 24\% possuíam uma faixa etária entre 26 e 30 anos; a maioria, $36 \%$, apresentava idade entre 31 e 45 anos e $8 \%$ deles estavam acima dos 45 anos. Em relação ao gênero, 64\% eram mulheres e $36 \%$ eram homens.

Nesse estudo foi encontrada uma prevalência significativamente maior de sinais e sintomas de DTM nas mulheres (64\%). Esses dados são similares aos encontrados na literatura. Britto ${ }^{16}$ ao avaliar alterações otológicas em portadores de desordens temporo-mandibulares que procuraram o serviço de DTM da Faculdade de Odontologia da UFRJ, concluiu que a frequência de DTM é maior no gênero feminino, sendo que $92,5 \%$ dos pacientes portadores de DTM que procuraram o serviço eram mulheres.

Algumas teorias têm tentado explicar por que as mulheres parecem ser mais afetadas do que os homens. Alguns autores ${ }^{17}$ acreditam que as mulheres não são tão aptas a lidar com suas pressões, o que resultaria num número maior de desordens funcionais, outros ${ }^{16}$ demonstram que os receptores de estrogênio na ATM das mulheres estão em maior quantidade.

Quanto a queixa ao abrir a boca, $40 \%$ dos pacientes afirmaram possuir dificuldades para abrir a boca, 36\% relataram sentir dor e desconforto, $24 \%$ possuíam algum tipo de estalido na articulação.Entre esses pacientes que possuíam estalido, $28,6 \%$ era do lado esquerdo, $14,3 \%$ do lado direito e $57,1 \%$ tinham presença de estalido em ambos os lados.A percentagem de pacientes que possuíam dor, desconforto e estalido na articulação foi de $32 \%$ e um número significativo $(36 \%)$ de pacientes possuía crepitação, onde $22,2 \%$ dessas crepitações eram do lado direito, $33,3 \%$ do lado esquerdo e $44,5 \%$ apresentavam de ambos os lados (Gráfico 1).

Os principais sinais e sintomas de DTM encontrados neste estudo foram: dor durante a mastigação, o bocejo e a fonação, dor de cabeça, dificuldade para abrir a boca, seguida de crepitação, desconforto e estalido. $\mathrm{Na}$ pesquisa de Malklin ${ }^{4}$ o sintoma mais prevalente foi o estalo (70\%), desvio dos movimentos mandibulares $(60 \%)$, dor de cabeça $(60 \%)$ e dor sobre a ATM (60\%). Nesse estudo constatou-se, em indivíduos com DTM, presença de desvio da mandíbula e redução vertical dos movimentos 


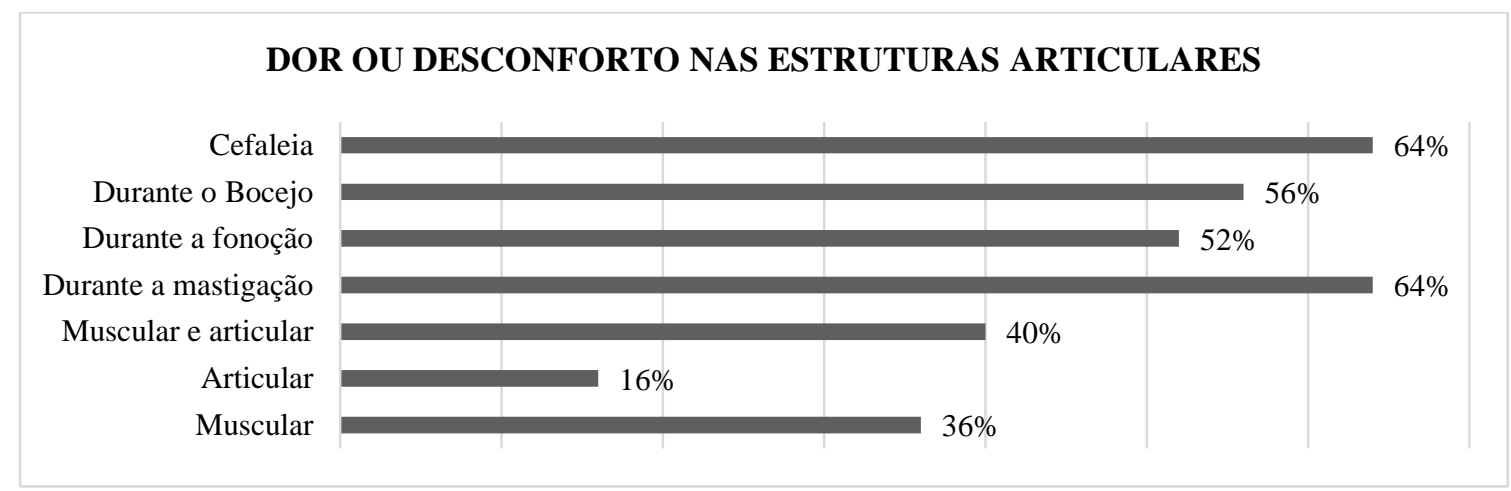

Gráfico 2: Gráfico referente à dor ou desconforto nas estruturas da articulação temporomandibular.

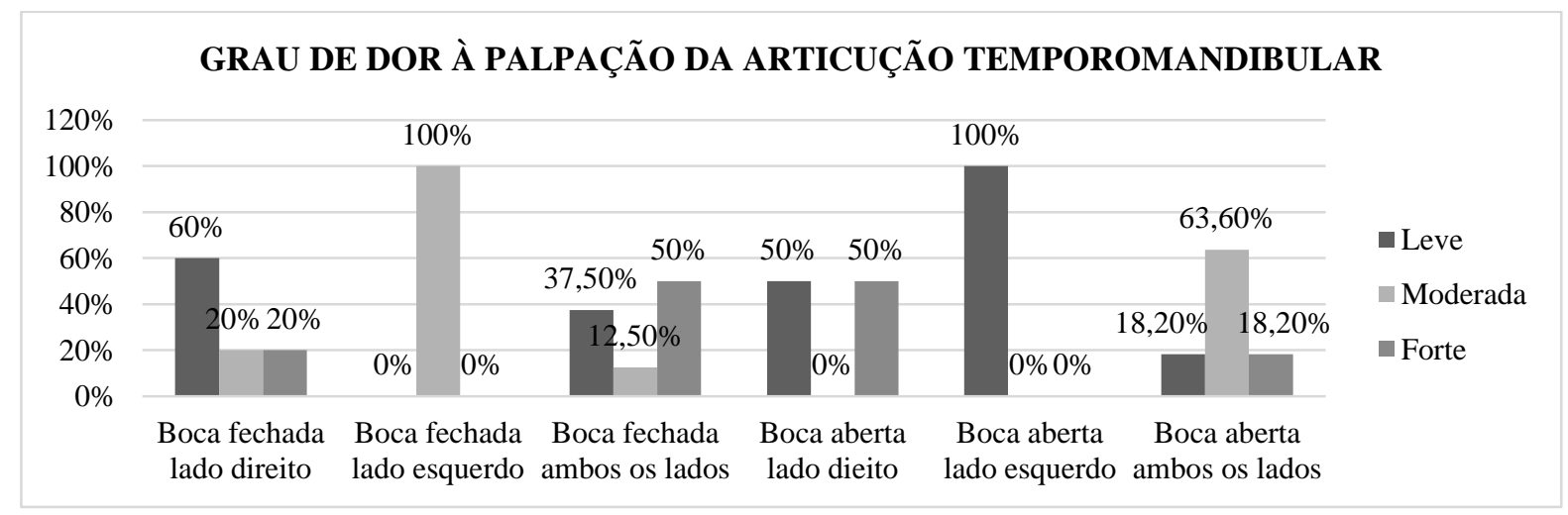

Gráfico 3: Distribuição percentual do grau de dor à palpação da articulação temporomandibular com a boca aberta e fechada.

Das 25 pessoas analisadas, portadoras de sinais e sintomas de DTM, 88\% apresentaram pelo menos um sintoma otológico. Entre os sintomas otológicos relatados pelos pacientes, a grande maioria, 72\%, afirmaram possuir zumbido; 56\% tinham otalgia; $52 \%$ apresentaram sensação de plenitude auricular; $52 \%$ dos pacientes relataram tontura com frequência, 44\% tinham hipoacusia e somente $12 \%$ desses indivíduos não possuíam nenhum tipo de sintoma (Gráfico 4).

Estes sintomas também têm sido relatados pela literatura em pacientes com DTM. Os sintomas otológicos estão associados com dificuldades nos movimentos mandibulares, abrir a boca e nas funções do sistema estomatognático (mastigação, deglutição, respiração e fala).É importante focar que se deve ficar atento ao sintoma de zumbido, visto que tal alteração pode ser decorrente de doenças da orelha interna ou sistema nervoso central e não apenas pela DTM. Pesquisa aponta que 0 côndilo da mandíbula ao sofrer um deslocamento pode provocar estiramento da cadeia ossicular e, assim, a ocorrência de sintomas otológicos $^{18}$. 


\section{SINTOMAS OTOLÓGICOS}

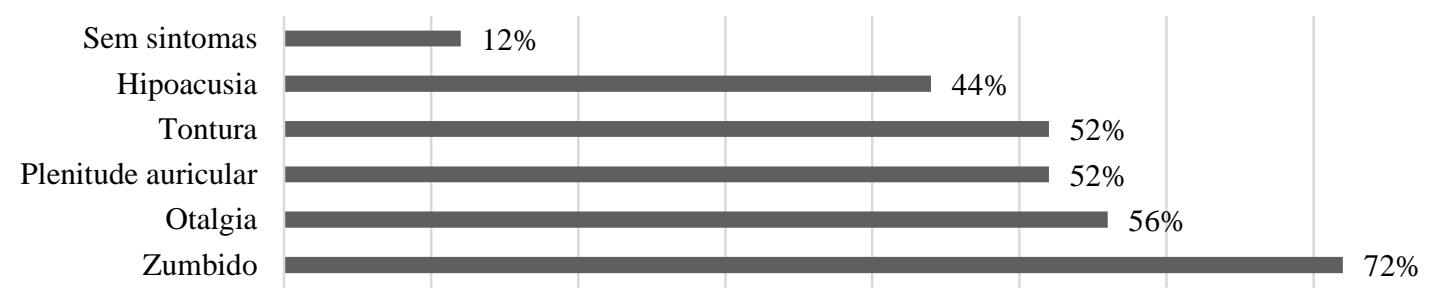

Gráfico 4: Sintomas auditivos presente nos pacientes analisados e incidência de cefaléia.

Outra hipótese vai na direção de apontar que a ocorrência de hiperatividade nos músculos da mastigação, por sua vez, levará a contração do músculo tensor de tímpano e da membrana timpânica ou contração muscular do véu palatino. Tal fato pode provocar disfunção da tuba auditiva e em sequência, sintomas de plenitude auricular, desequilíbrio e perda de audição ${ }^{16}$.Pode-se dizer que a relação e origem dos sintomas otológicos ainda não foram totalmente esclarecidas. Porém, pesquisa comprova que as DTMs, geralmente, vêm associadas a sintomas otológicos ${ }^{18}$.

Queixa de otalgia em paciente com DTM pode ser dor referida, cuja origem pode estar em algum músculo com hiperatividade, ou da própria ATM. Mioespasmo do músculo tensor da membrana do tímpano pode ser um efeito secundário como resposta à dor gerada pela DTM, podendo tracionar o martelo e a membrana timpânica, alterando a condução do som através da orelha média, o que justificaria a queixa de hipoacusia subjetiva e também de otalgia ${ }^{18}$.

Quanto aos achados dos exames audiométricos dos pacientes que possuíam sinais e sintomas sugestivos de disfunção temporomandibular, 56\% desses pacientes não possuíam perda auditiva; $12 \%$ apresentavam uma perda auditiva de grau leve; $12 \%$, de grau moderado, somente $4 \%$ de grau severo e $16 \%$ de grau profundo. Em relação ao tipo de perda, o mais prevalente foi sensorioneural, com $54.6 \%$; seguido do tipo condutiva com $36,4 \%$, e $9 \%$ era de perdas do tipo mista.

De acordo com os resultados do Índice Percentual de Reconhecimento de Fala (IPRF) $72 \%$ dos indivíduos possuíram um reconhecimento entre 92 e $100 \%$ das palavras apresentadas. $68 \%$ apresentaram curvas timpanométricas do tipo "A", característico de normalidade, $16 \%$ tinham curva do tipo "C" e $12 \%$ possuíam curva do tipo "Ad". Quanto aos resultado dos Reflexos Acústicos Contralateral em $60 \%$ dos pacientes estava presente (Tabela 1).

Quanto aos achados nos exames audiológicos, a grande maioria dos pacientes encontrou-se dentro do padrão da normalidade. Entretanto $44 \%$ deles possuíam algum tipo de perda auditiva. De acordo com a pesquisa de Felício $^{9}$ os resultados da audiometria, na orelha direita as médias dos limiares de via aérea nas frequências de 250, 500, 1000, 2000 e $4000 \mathrm{~Hz}$ apenas 3 sujeitos apresentaram médias acima de 20 
dBNA. Na orelha esquerda as médias dos limiares de via aérea nas mesmas frequênciasapenas 1 sujeito apresentou média acima de 20 dBNA. Nenhum sujeito apresentou índice de reconhecimento de fala abaixo de $92 \%$.

$\mathrm{Na}$ amostra de Felício $^{9}$ verificouse na timpanometria a curva tipo $\mathrm{A}$ em 48 orelhas, a tipo Ad em 3 orelhas direitas e 2 esquerdas e a tipo $\mathrm{C}$ em uma orelha esquerda. Portanto, a curva tipo A prevaleceu de modo significante $\quad(p<0,01) . \quad O \quad$ reflexo estapediano ipsi e contralateral esteve presente com maior significância estatística em todas as frequências analisadas e em ambas as orelhas, isto é, em 22 dos 27 sujeitos. Esses resultados corroboram com os dados encontrados na atual pesquisa.

Assim, a desarmonia do sistema estomatognático, que se manifesta como dor orofacial e dificuldade nas atividades funcionais foram associadas de modo significante aos sintomas otológicos nos casos de DTM

Tabela 1: Percentual dos dados encontrados nos exames audiométricos realizados com os pacientes.

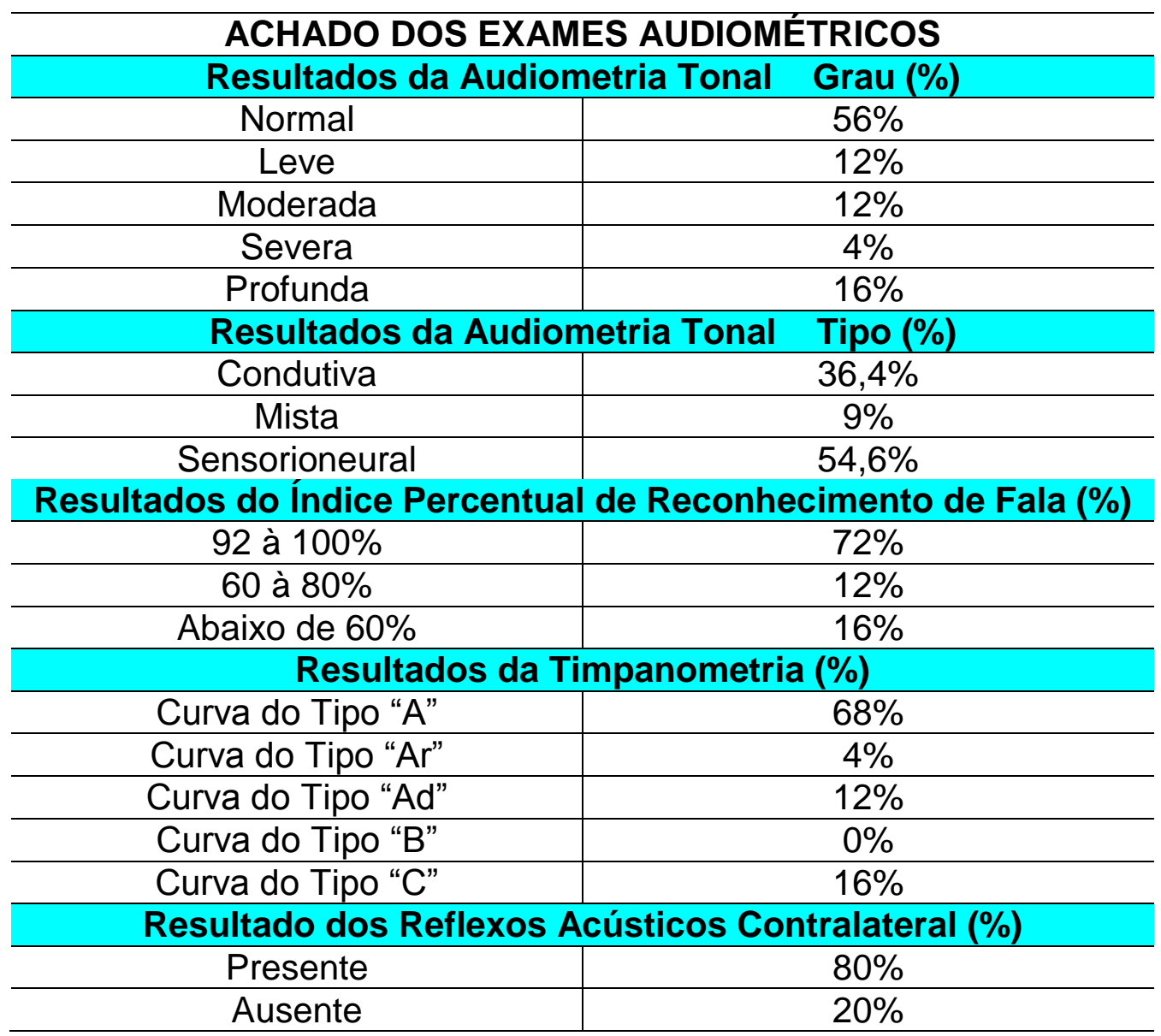




\section{Conclusão}

Com base nos resultados pôdese concluir que a amostra estudada apresentou uma alta prevalência de sintomas otológicos, prevalecendo o zumbido, a otalgia a plenitude auricular, além de outros sinais e sintomas relacionados à DTM. A prevalência de resultados dentro dos padrões de normalidade nos exames otológicos e audiológicos sugeriu que os sintomas otológicos não refletiam as condições das orelhas, verificadas no exame clínico audiológico.

Os achados deste estudo apontam alto percentualde sintomas otológicos em pacientes com sinais e sintomas de DTM. Destaca-se a importância da pesquisa dos sintomas otológicos na avaliação das DTMs. Entretanto há a necessidade de estudos e pesquisas mais complexos e com uma amostra maior pra comprovar as específicas relações causa-efeito entre ambos, direcionando a elaboração de diagnósticos e tratamentos mais precisos para a promoção da saúde do aparelho estomatognático.

\section{Referências}

1. Felicio CM. Alterações auditivas relacionadas ao zumbido nos distúrbios otológicos e da articulação temporomandibular. Revista Brasileira de Otorrinolaringologia, v.75, n.2, 14146, mar/abr. 2012.

2. Meira GSP. DTM $x$ Problemas Otológicos. Rev AONP online [periódico na Internet]. Out/Nov, 2011 [acesso em: 12 maio 2013]; (7).

Disponível em:http://www.aonp.org.br/fso/revi sta7/rev712a.htm.
3. Maciel RN. Oclusão e ATM Procedimentos clínicos. São Paulo: Editora Santos, 1998.

4. Malklin DP. The role of TMJ dysfunction in the etiology of middle ear disease. International Journal Orthodontics, v.25, n.1, p. 20-1. 2007.

5. Manfredi APS; Vendite LL. Avaliação de sensibilidade do questionário de triagem para dor orofacial e desordens temporomandibulares recomendado pela Academia Americana de Dor Orofacial. Revista Brasileira de Otorrinolaringologia, v. 67, ก. 6, p.763-8, nov./dez. 2009.

6. Penkner K, Kole W, Kainz J, Shied $\mathrm{G}$, Lorenzoni M. The function of tensor velipalatini muscles in pacients with aural symptoms and temporomandibular disorder. An EMG study. Journal of Oral Rehabilitation, v.2, n.4, p.3448. 2010.

7. Molina O. F. Fisiopatologia craniomandibular - oclusão e ATM. 2. ed.São Paulo: Pancast, 1995.

8. BianchiniEMG. Articulação temporomandibular: implicações, limitações e possibilidades fonoaudiológicas. $2 . \quad$ ed. Carapicuíba: Pró-Fono, 2010.

9. Felício CM; Faria TG, Silva MAMR, Aquino AMCM; Junqueira CAJ. Desordem temporomandibular: relações entre sintomas otológicos e orofaciais. Revista Brasileira de Otorrinolaringologia, v. 70 , ก. 6 , p.786-93, nov./dez. 2008.

10. Russo ICP, Santos TMM. Prática da audiologia clínica. São Paulo: Cortez, 2009.

11. Celic R, Jerolimov V, Filipovié-Zore I, Knezovic-Zlataric DD. The prevalence of temporomandibular disorders in a non-patient 
population. Acta Stomatol Croat, v. 35, n. 3, p. 327-30, may. 2011.

12. Fonseca DM, Bonfante G, Valle AL, Freitas SFT. Diagnóstico pela anamnese da disfunção craniomandibular. Revista Gaucha de Odontologia, v. 32, 23-8. 2008.

13. Maciel RN. ATM e dores craniofaciais: fisiopatologia básica. São Paulo: Editora Santos, 2003.

14. Gracely RH, Reid KI. Mensuração da dor orofacial. In: FRICTON, J.R.; DUBNER, R. Dor orofacial e desordens temporomandibulares. São Paulo: Editora Santos, 2003. pg. 117-43.

15. Michelotti A, Steenks HM, Farella M, Parisini F, Cimino R, Martina R. The Additional Value of a Home Physical Therapy Regimen Versus Patient Education Only for the Treatment of Myofascial Pain of the Jaw Muscles: Short-term Results of a Randomized Clinical Trial. Journal Orofacial Pain, v. 18, n. 2, p.114-125, mar./apr. 2004.

16. Britto LH. Alterações otológicas nas desordens temporomandibulares. Rev Bras Otorrinolaringol 2005; 1-75.

17. Agerberg G, Carlsson GE. Symptoms of functional disturbances of the masticatory system. A comparison of frequencies in a population sample and in a group of patients. Acta Odontol Scand 2004;33(4):183-90.

18. Mota LAA, Albuquerque KMG, Santos MHP, TravassosRO. Sinais e sintomas associados à otalgia na disfunção temporomandibular. Arq Int Otorrinolaringol. 2007, 11(4): 411-415. 